\title{
Démarcation des terres indigènes et inégalités en zone de front pionnier au Nicaragua: le cas de Tasba Pri
}

\author{
Florent Leo ${ }^{1,2, *}$ et Nadège Garambois ${ }^{3}$ \\ ${ }^{1}$ EHESS, FD territoires, sociétés et développement, 190-198 avenue de France, 75013 Paris, France \\ 2 BICU, UFR Ing. agricole et forestière, 71000 Bilwi, Nicaragua \\ 3 AgroParisTech, UFR agriculture comparée et développement agricole, UMR Prodig, 16 rue Claude-Bernard, 75005 Paris, France
}

\begin{abstract}
Résumé - Le nord de la côte atlantique du Nicaragua, initialement peuplé d'indigènes, principalement Miskitus, est longtemps resté forestier et en marge du cœur économique du pays. Depuis les années 1950, il connaît l'avancée d'un véritable front pionnier de populations métisses en provenance de la moitié pacifique du pays, en quête de terres à défricher pour développer des élevages bovins. Après avoir marqué le pas durant la période sandiniste (1979-1990), la progression de ce front s'accélère depuis le début des années 1990 et contribue au fait qu'aujourd'hui, seulement $45 \%$ de la population de la Région autonome de la Côte caraïbe nord (RACCN) soit d'affiliation miskitue. À Tasba Pri, l'arrivée continue d'éleveurs bovins sur les terres communales et l'intégration progressive aux échanges marchands se sont le plus souvent accompagnées de l'éviction d'une partie des familles miskitues de leurs terres et de la captation d'une rente foncière par leurs élites. Le creusement des inégalités inter- et intra-ethniques conduit à une fracture profonde entre systèmes familiaux précarisés et systèmes patronaux, marquée par la multiplication des violences autour de l'accès au foncier. Instrumentalisés par une partie des élites indigènes locales, la législation foncière spécifique à la démarcation des territoires indigènes (loi 445 de 2003) ne contribue pas à régler ces conflits, mais à donner un semblant de légalité à la vente de terres communales.
\end{abstract}

Mots clés : front pionnier agricole / accaparement foncier / territoire indigène / agriculture comparée / Nicaragua

\begin{abstract}
Demarcation of indigenous lands and inequalities in pioneer front area in Nicaragua: the case of Tasba Pri. The northern part of the Nicaraguan Atlantic Coast, originally populated mostly by indigenous people, mainly Miskitu, has remained forested and isolated from the country's economic heart for a long time. In the 1950s, the region experienced an increase in pioneer migration from the pacific part of the country in search of land to develop cattle breeding. After stagnating during the Sandinista period (1979-1990), the progress of the pioneer front accelerated again in the early 1990s and partially explains why only $45 \%$ of the population in the North Caribbean Coast Autonomous Region (NCCAR) are of Miskitu origin today. In Tasba Pri, the continuous flow of livestock farmers on communal land and the gradual integration of this territory into the commercial exchanges were most often accompanied by the eviction of a part of Miskitu families from their lands and the capture of a land rent by their elites. The widening of inter- and intra-ethnic inequalities has led to a deep divide between precarious family systems and large-scale family systems, marked by the increase of violence related to land access. Used by part of the local indigenous elites, land legislation on the demarcation of indigenous territories (law 445 of 2003) does not resolve these conflicts but just gives a semblance of legality to the sale of communal land.
\end{abstract}

Keywords: agricultural pioneer front / land grabbing / indigenous territory / compared agriculture / Nicaragua

\section{Introduction}

La Région autonome de la Côte caraïbe nord (RACCN), occupée à l'origine par des populations indigènes, enclavée par rapport au cœur économique du Nicaragua, longtemps centrée

\footnotetext{
$\overline{* \text { Auteur de correspondance }}$ : florent.leo@agroparistech.fr
}

sur l'exploitation des ressources forestières, minières, halieutiques - et des hommes - par des compagnies étrangères, est restée, jusqu'à la révolution sandiniste, assez largement en marge du reste du pays (Boege, 1987). Représentant plus de $25 \%$ de la superficie du Nicaragua, cette région regroupe aujourd'hui encore moins de $5 \%$ de sa population. La RACCN (Fig. 1) enregistrait ainsi en 2012 une densité de population de 


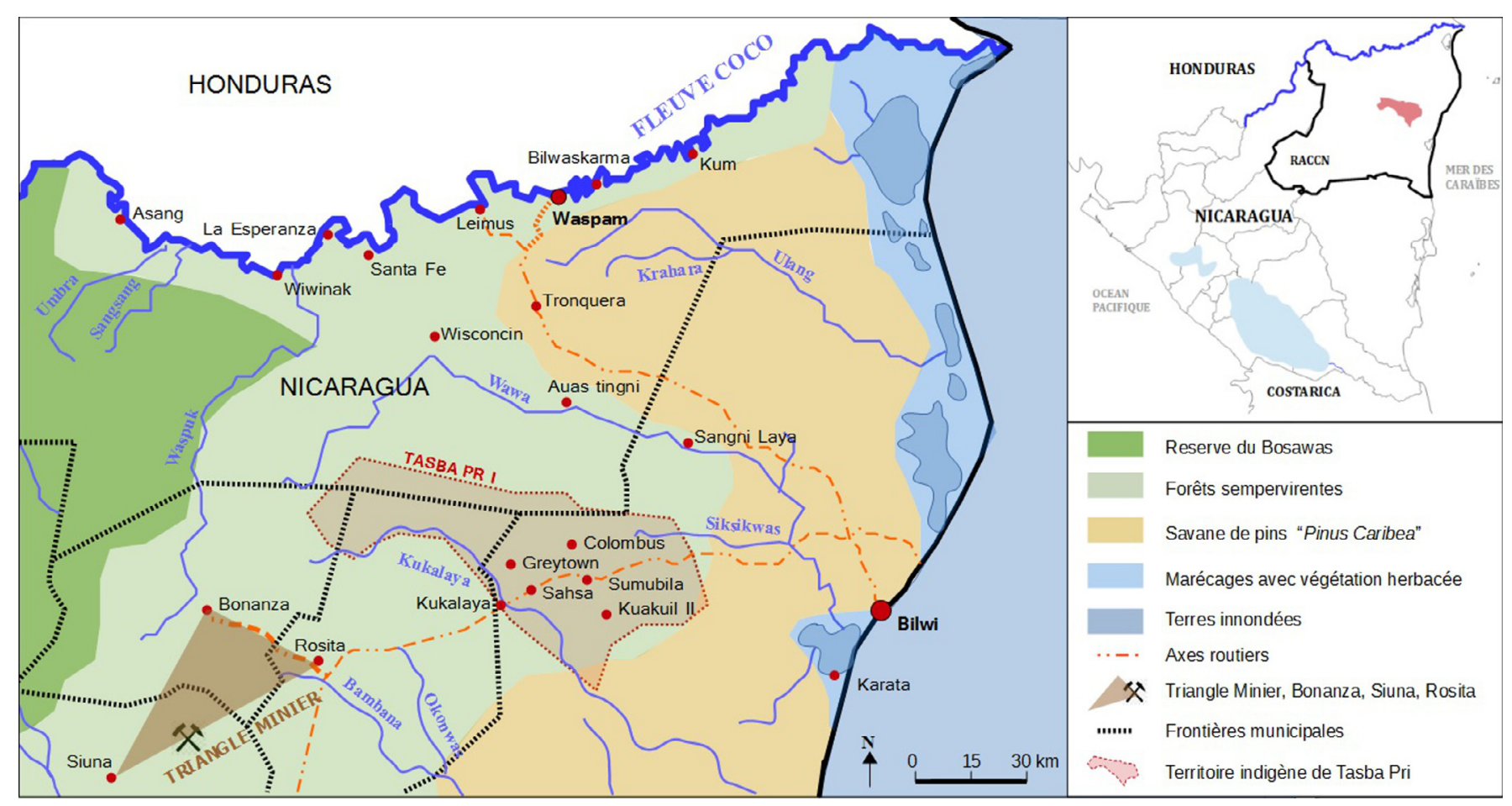

Fig. 1. Le territoire indigène de Tasba Pri au sein de la RACCN. Réalisation: Leo Florent.

Fig. 1. The indigenous territory of Tasba Pri within the NCCAR.

moins de 15 habitants au $\mathrm{km}^{2}$, mais qui a plus que doublé depuis 1995 (Fondation pour le développement technologique agricole et forestier du Nicaragua [FUNICA], 2009). Initialement peuplée en majorité par des indigènes Miskitus (et dans une moindre mesure Mayangnas), la RACCN a connu, depuis la chute du gouvernement sandiniste en 1990, d'importants flux migratoires en provenance des régions plus peuplées du Nicaragua Pacifique (Hardy, 2005). Ces flux alimentent un véritable front pionnier dans les zones forestières intérieures, conduisant à son déboisement progressif au profit du développement de l'élevage bovin et à l'installation de familles sans affiliation indigène, qualifiées de «métisses», toujours plus près des finages des villages indigènes. $\mathrm{Si}$ les Miskitus constituent l'ethnie indigène majoritaire (84\%) (FUNICA, 2009), ils représentent aujourd'hui à peine $45 \%$ de la population totale de la RACCN.

La RACCN dispose depuis 1987 d'un régime d'exception qui acte son autonomie dans la gestion de ses ressources et dans son organisation sociale et administrative. Dès la fin des années 1980, cette législation spécifique déclarait toutes les terres de la région de statut «communal» et «inaliénable», sans définir pour autant selon quelles modalités cette incessibilité pouvait s'exercer. Une loi additionnelle (loi 445) sur la propriété communale des peuples indigènes des régions autonomes de la côte atlantique a été promulguée en 2003. Elle vise à régulariser la propriété communale en procédant à la démarcation précise et à la titularisation de «territoires indigènes », puis en mettant en place un processus que le texte de loi qualifie d' «assainissement» (saneamiento), afin d'écarter les tiers de ces territoires déclarés «indigènes », mais dans les faits aujourd'hui pluriethniques.
Ce texte présente l'impact de ce front pionnier sur l'agriculture pratiquée dans la région forestière de Tasba Pri (Fig. 1) et sur les populations indigènes le peuplant, ainsi que les effets de l'application concrète de la loi 445. Situé le long de l'axe routier Bilwi-Rosita, Tasba Pri est l'un des 19 territoires indigènes de la RACCN délimités dans le cadre de l'application de la loi 445 , et celui qui présente la plus faible proportion de population indigène (16\% en 2008). Il connaît des conflits fonciers et des violences interethniques qui s'intensifient depuis qu'a débuté la dernière étape de l'application de la loi 445, dite d'«assainissement ».

\section{Méthodes}

Cette recherche s'appuie sur un «diagnostic agraire» (Cochet et Devienne, 2006) conduit au cœur des communautés $\mathrm{du}$ «territoire indigène de Tasba Pri ». Ce travail de terrain de cinq mois a reposé sur l'étude des dynamiques agraires de cette petite région (Leo, 2016) au travers de:

- l'analyse de l'évolution des modes d'utilisation du milieu en lien avec les processus de différenciation des systèmes de production ;

- l'identification sur cette base d'une typologie des systèmes de production agricoles actuels ;

- la modélisation et comparaison du fonctionnement technique et des résultats économiques des différents «systèmes de production» agricoles (Cochet et al., 2007) identifiés et des «systèmes d'activité » (Paul et al., 1994) qui leur correspondent. 
Ce travail s'est notamment appuyé sur une cinquantaine d'enquêtes historiques auprès d'agriculteurs âgés, témoins et acteurs des transformations passées et sur une cinquantaine d'enquêtes technico-économiques approfondies auprès d'agriculteurs de la région en activité. Celles-ci ont été conduites sur la base d'un échantillonnage «raisonné », qui ne vise pas à la représentativité statistique, mais à pouvoir illustrer de façon archétypale les différentes trajectoires d'évolution et les systèmes de production actuels.

\section{Le territoire indigène de Tasba Pri, une région forestière de peuplement récent}

\subsection{Une région d'installation subie dans les années 1980}

Le front pionnier de colonisation de la moitié atlantique à partir du sud-ouest, avec l'installation de grandes exploitations d'élevage, a débuté dès les années 1950 au Nicaragua (Lévêque, 1986), mais s'est vu stoppé durant la période sandiniste (1979-1989). Avant les années 1990, Tasba Pri n'a été que marginalement atteinte par le front pionnier, avec la création, à la toute fin des années 1970, de la route reliant les régions minières forestières au littoral atlantique (Fig. 1). Elle n'a connu que de rares sédentarisations de familles miskitues chassées de leur village d'origine par l'implantation d'industries étrangères d'exploitation du bois (Fig. 2a). Elle a été en revanche le lieu d'installation forcée de 8500 Miskitus en 1982 par le gouvernement sandiniste (Kinlochet Tijerno, 1997 ; Klein, 1986), qui cherchait à fragiliser l'opposition miskitue qui s'était dressée contre lui dans cette partie du pays. Les familles ont été réparties dans cinq réserves (Sahsa, Kukalaya, Colombus, Wasminona et Truslaya) qui s'apparentaient davantage à des camps, tandis qu'étaient expulsées les quelques familles miskitues qui s'étaient installées avant cela dans la région (Fig. 2b).

\subsection{De 1990 à 2007 : reprise du front pionnier agricole depuis l'ouest du Nicaragua}

La loi votée par le gouvernement sandiniste en 1987 afin d'assouplir les relations avec les populations indigènes, relative à l'autonomie des régions de la côte atlantique (loi $\mathrm{n}^{\mathrm{o}} 28$ ), prévoit que «les terres, eaux et forêts qui ont traditionnellement appartenu aux communautés de la côte atlantique constituent la propriété communale» et les déclare « inaliénables » et « imprescriptibles ». Elle n'a pas le temps de se traduire en effets avant le renversement du gouvernement sandiniste en 1990.

Dès la loi de 1987, puis de façon accélérée à partir de 1990, les cinq camps se vident : la majorité des familles miskitues retournent dans leurs villages d'origine le long du Río Coco. $\mathrm{Ne}$ demeurent que de rares familles miskitues, souvent celles qui s'étaient liées sur place avec les représentants sandinistes responsables des camps. Celles-ci fondent des villages sur le finage des anciens camps situés en milieu de versant (Sahsa et Sumubila) à proximité de la route. Chaque village abrite une quinzaine de familles miskitues auxquelles est attribué, à la faveur de l'application de la loi $\mathrm{n}^{\mathrm{o}} 28$ de 1987, un titre communal collectif d'environ 4000 ha sous forme de titre de réforme agraire. Les familles miskitues arrivées dans la région dès les années 1960, déplacées pendant la période sandiniste, se réinstallent à la même époque en créant leurs propres villages (Kuakuil II et Altamira). Elles disposent également d'un titre communal de réforme agraire de 1500 à 2000 ha pour une dizaine de familles. Ces surfaces dépassent largement les besoins et la capacité de production des familles à l'époque, qui cultivent chacune environ 15 ha en abattis-brûlis avec un outillage strictement manuel.

Les régions atlantiques font alors figure d'espace libre (Hardy, 2005) pour les nombreux agriculteurs métis de la moitié ouest du pays fuyant pauvreté et accès précaire au foncier (Bainville et al., 2005). Ils viennent tenter leur chance et contribuent à relancer dès le début des années 1990 l'avancée du front pionnier depuis le sud-ouest vers le nord-est de la région atlantique, sans intervention de l'État pour organiser et canaliser ces flux. Un triple mouvement migratoire s'opère alors dans la région de Tasba Pri. Des familles miskitues venant du sud-ouest de la zone forestière, chassées par l'installation de familles métisses sur leur finage, migrent dans les villages fondés par des Miskitus sur les anciens camps et accèdent à des terres sans pour autant figurer sur le titre de propriété communale comme familles membres du village. Des familles métisses natives de la RACCN, disposant d'un capital de départ suffisant, s'installent au sein des finages des villages miskitus, en milieu de versant et à proximité de la route et des infrastructures, sans titre de propriété légale mais en achetant chacune aux autorités villageoises un lot pouvant atteindre jusqu'à 35 ha de forêt, prélevé sur la propriété communale. En une dizaine d'années, ils parviennent à convertir ces terres en pâturages et à accumuler suffisamment de capital pour accroître leur cheptel et s'agrandir, en achetant progressivement, toujours sans titre de propriété, des lots supplémentaires de 3 à 5 ha aux autorités indigènes. Enfin, dans le sillage d'entreprises forestières, des familles métisses issues du centre-ouest, plus pauvres et sans moyens suffisants pour acheter des terres à proximité de la route (et des finages des villages miskitus), s'installent avec quelques vaches sur les hauts de versant, sans titre de propriété, et défrichent progressivement des parcelles qu'elles clôturent et convertissent en prairies permanentes (Fig. 2c).

\subsection{Depuis la fin des années 2000, des inégalités croissantes dans l'accès au foncier}

Durement touchés par l'ouragan Felix en septembre 2007, nombre d'éleveurs métis installés en haut de versant préfèrent alors migrer plus au nord (vers des terres encore boisées) avec ce qu'il leur reste de troupeau. Ils vendent les terres qu'ils avaient défrichées et aménagées à de riches familles d'éleveurs du centre du Nicaragua, en quête de nouveaux espaces où la disponibilité fourragère est régulière tout au long de l'année. Les vendeurs ne disposant pas de titre de propriété, ces transactions sont matérialisées par un simple acte de vente sans valeur légale. Une dizaine d'exploitations d'élevage bovin à salariés se sont ainsi créées dans la région, disposant chacune d'un à trois lots de 150 à 200 ha, contribuant à renforcer encore la concentration foncière.

Face aux agrandissements des exploitations des premiers éleveurs métis installés et au flux continu de migrants métis 

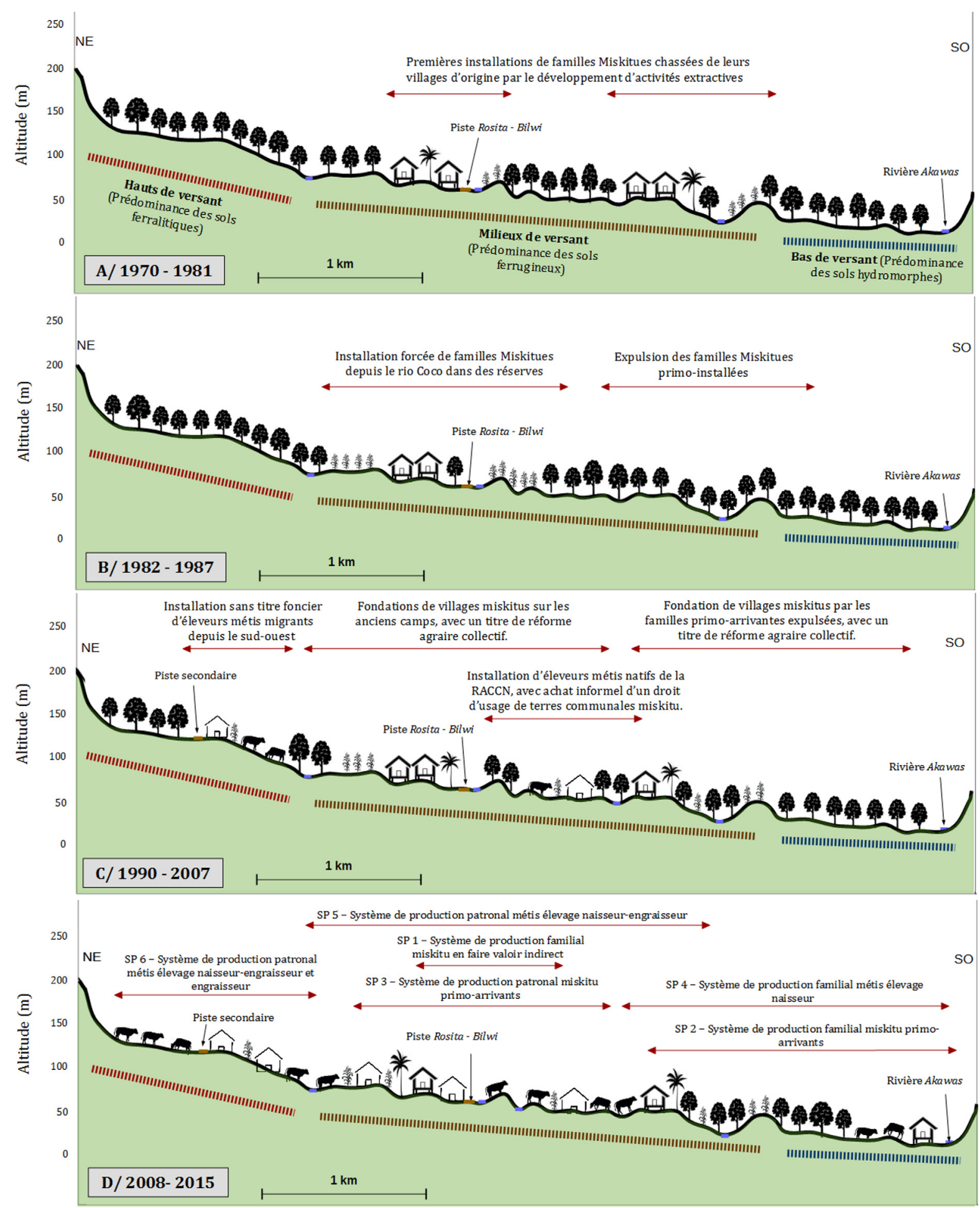

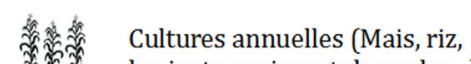
haricots, racines, tubercules...)

Elevage bovin (pâturages)
Forêts sempervirentes

* Arbres fruitiers (et musaceas comme plantes pluriannuelles..) 国
Habitations de familles métisses

Habitations de familles miskitues

Fig. 2. Occupation progressive des différents étages écologiques de la région de Tasba Pri et localisation des différents systèmes de production agricoles actuels. Réalisation: Leo Florent; source : enquêtes.

Fig. 2. progressive occupation of the different ecological stages of the Tasba Pri region and location of the different current agricultural production systems. 
dans la région, les réserves de terres communales indigènes en milieu de versant sont, dans la majorité des villages, toutes vendues dès la fin des années 2000. La poursuite de ce processus, qui intéresse à la fois les élites villageoises miskitues détournant pour leur compte cette rente foncière et les éleveurs métis, supposait dès lors de s'attaquer aux terres des Miskitus arrivés dans un second temps au sein des villages, qui ne figurent pas dans la liste officielle des familles fondatrices et ne sont pas protégés par la loi de 1987 relative à la propriété communale. Expulsées des terres qu'elles exploitaient jusqu'alors, ces familles indigènes en sont réduites à prendre en fermage moins de trois hectares, dont l'accès est souvent assorti de corvées. Les familles indigènes primoarrivantes conservent pour la plupart 15 à 20 ha mais ne disposent plus, dans la majorité des villages, de réserves de terres communales suffisantes pour installer leurs enfants, qui sont aujourd'hui nombreux à migrer vers les centres urbains. Actuellement à la tête de systèmes patronaux déployés sur plus de 100 ha en moyenne, les familles de l'élite politique indigène ont su quant à elles se doter d'un pouvoir économique croissant, en se réservant de vastes superficies, en investissant grâce au capital accumulé par la vente des terres communales et en étant les mieux placées pour profiter de l'aide internationale et des projets de développement initiés dans la région à la suite de l'ouragan Felix de 2007.

Les migrants métis les plus pauvres qui continuent à affluer dans la région s'installent depuis quelques années en rachetant de petites surfaces en milieu de versant à des primo-défricheurs désirant partir plus au nord vers des terres encore inoccupées, mais le plus souvent en achetant aux autorités indigènes des terres de bas de versant ( 15 à $25 \mathrm{ha}$ ), en partie inondables et donc bien moins coûteuses (Fig. 2d).

\section{Une différenciation sociale aujourd'hui profonde entre producteurs métis comme indigènes}

Sans ethniciser a priori les catégories de producteurs dans cette recherche, l'étude de la différenciation des systèmes de production a conduit à proposer une typologie des systèmes actuels qui coïncide de fait avec l'affiliation «indigène» ou «métisse » des familles qui les mettent en œuvre, car celle-ci a contribué à déterminer tout au long de l'histoire la localisation de ces exploitations, leur accès au foncier, au capital et au travail, et leur intégration dans les échanges marchands.

Alors qu'elles cultivaient des surfaces assez semblables il y a moins de 30 ans, une profonde différenciation sociale s'observe aujourd'hui au sein des familles miskitues. Les familles miskitues les plus pauvres, en fermage sur quelques hectares qui suffisent à peine à assurer leur autoconsommation (système de production 1 [SP1]), vivent avec 700 à 1550 euros de revenu agricole par actif familial et par an. À titre de comparaison, le revenu annuel d'un salarié agricole à temps plein à Tasba Pri est de 1100 euros. Considérant que, dans la région, un actif a besoin d'au moins 1500 euros par an pour couvrir ses besoins incompressibles et ceux de ses dépendants, ces familles ne survivent que grâce à des activités complémentaires de pêche et en s'embauchant comme journaliers agricoles chez les familles miskitues les plus riches ou par une migration de travail saisonnière à Puerto
Cabezas (de plus en plus souvent définitive pour les jeunes actifs). La plupart des familles miskitues primoarrivantes conduisent encore sur 15 à 20 ha une agriculture de polyculture - élevage basée sur l'abattis - brûlis, centrée sur la production de riz, de maïs et de haricot pour l'autoconsommation et sur la production de manioc afin d'engraisser des porcs destinés à la vente (SP2). Elles dégagent un revenu agricole de 1400 à 2200 euros par actif familial et par an. Parmi ces familles fondatrices, les élites politiques villageoises ont souvent su se réserver un droit d'usage sur 100 à 250 ha, dont elles n'exploitent réellement que la moitié, pour mettre en œuvre un système patronal (c'est-à-dire où la main-d'œuvre familiale est complétée d'un recours structurel au travail salarié). Elles disposent d'un revenu agricole de 6700 à 10700 euros par actif familial et par an en combinant cultures vivrières, culture de rente et - seules familles miskitues à en disposer - élevage bovin (SP3).

Toutes centrées sur l'élevage bovin et disposant comparativement de surfaces supérieures, les familles métisses enregistrent néanmoins des écarts de revenus importants entre elles, liés à leur date d'arrivée dans la région et au capital dont elles disposaient alors, qui commandent l'étage écologique où elles ont pu s'installer, la qualité de leurs pâturages et l'ampleur de leur capitalisation dans le bétail et la terre, selon des mécanismes proches de ceux décrits par Maldidier (2004). Les agriculteurs arrivés dans la région depuis moins de dix ans mettent en œuvre un système familial sur 25 à 40 ha en bas de versant. Ils ne disposent pas de prairies suffisamment productives (prédominance de la graminée invasive retana [Ischae munindicum]) pour engraisser leurs bovins et combinent un élevage bovin naisseur de 5 à 15 mères avec un élevage de porcs engraissés au manioc et des cultures de riz, maïs et haricot en partie destinées à la vente (SP4), pour un revenu agricole de 1600 à 2700 euros par actif familial et par an. En dessous de 25 ha, ils travaillent en complément comme journaliers agricoles chez les éleveurs métis plus riches en attendant de pouvoir s'agrandir. Disposant de terres à plus haut potentiel et plus avancées dans le processus de capitalisation, les familles métisses arrivées plus tôt et installées en milieu de versant conduisent des systèmes patronaux sur 70 à 100 ha. Elles ont les moyens d'engraisser leurs animaux grâce à leurs prairies temporaires de Brachiaria brizantha ou de Panicum maximum et disposent d'un revenu agricole de 8000 à 12000 euros par actif familial et par an (SP5). Les plus vastes troupeaux (75 à 125 mères) sont aux mains de riches éleveurs patronaux qui ont progressivement constitué des lots de 150 à 200 ha (SP6), qu'ils confient à un contremaître ou à un de leur fils, secondé de salariés, pour y conduire un élevage bovin naisseurengraisseur, complété de l'engraissement des mâles achetés aux éleveurs naisseurs de la région, pour un revenu agricole de 7000 à 10000 euros par actif familial et par an.

Toutes les familles complètent ces revenus agricoles avec des activités extra-agricoles, essentielles à la survie des familles miskitues les plus pauvres et facteur d'accélération de la capitalisation pour les éleveurs métis disposant de plus faibles surfaces, mais qui ne parviennent pas à infléchir les inégalités sociales. Les familles les plus pauvres en sont réduites à vendre leur force de travail, tandis que les plus riches développent des activités de négoce grâce au capital accumulé dans l'agriculture. Exprimés par actif familial, les écarts de 
revenus enregistrés atteignent ainsi un facteur de 10 à 15 pour le revenu agricole comme pour le revenu total, avec une dualité profonde, non pas entre systèmes métis et systèmes miskitus, mais bien entre systèmes familiaux, d'une part (miskitus comme métis), et systèmes patronaux métis et miskitu, d'autre part.

Parallèlement, des évictions foncières menées à plus grande échelle par des entreprises extractives ont conduit des communautés indigènes à déposer une plainte devant la Cour interaméricaine des droits de l'homme de l'Organisation des États américains. Celle-ci a imposé, en retour, à l'État nicaraguayen de légiférer sur le statut des terres indigènes, conduisant en 2003 à la publication de la loi 445. L'application depuis 2007 de cette loi qui prévoit, dans les régions de la côte atlantique du Nicaragua, d'écarter les tiers non autochtones des territoires déclarés «indigènes », mais où les familles d'affiliation «métisse » dominent largement en nombre, intervient à Tasba Pri dans un contexte d'inégalités sociales explosif.

\section{La loi 445 : un outil susceptible de réduire les inégalités et d'apaiser les conflits ?}

\subsection{L'esprit de la loi 445}

Afin de régulariser le statut de la propriété communale des terres indigènes, la loi 445 prévoit un processus en cinq étapes (chapitre VIII):

- la demande de démarcation des finages des communautés indigènes ;

- la résolution des conflits internes qui surviendraient lors de ce processus de délimitation ;

- la mesure et le bornage des terres revendiquées par les communautés ;

- la titularisation des terres ;

- l'«assainissement » (saneamiento) des terres au regard des tiers qui s'y trouveraient installés, personnes naturelles ou juridiques entendues comme «extérieures aux communautés et proclamant un droit de propriété sur des terres communales » (article 3$)$.

Pour ce faire, la loi 445 acte la création et l'institutionnalisation du rôle des autorités communales et de l'autorité territoriale (article 4), ainsi que des modalités de leur élection (article 7). Elle prévoit la possibilité pour les autorités communales «d'octroyer des autorisations pour disposer des terres communales et des ressources naturelles en faveur de tiers, à condition d'être mandatées expressément par l'Assemblée communale » (article 10), ainsi que «l'administration des droits de propriété communale par l'autorité territoriale et les autorités communales » (article 30$)$. Sont créés à cette fin la Commission nationale de démarcation et de titularisation (Conadeti) et l'Office de titularisation rurale (OTR) en charge d'émettre les titres (article 43).

La loi 445 comporte également plusieurs articles relatifs au devenir des tiers qui exploitent des terres situées sur les propriétés communales indigènes sans appartenir à ces communautés. Ainsi, «un tiers détenteur d'un titre agraire sur des terres indigènes a le droit de continuer à la posséder » mais « ne pourra prétendre l'aliéner et devra alors vendre les 'améliorations' [qu'il a effectuées] à la communauté » en lui rétrocédant la terre (article 36) ; «un tiers détenteur d'un titre agraire présentant un vice de forme ou de fond sur des terres indigènes sera indemnisé pour que celles-ci soient rétrocédées à la communauté indigène concernée » (article 37) ; tandis qu'un «tiers sans aucun titre devra abandonner les terres indigènes sans indemnisation » ou devra «payer un loyer à la communauté » au cas où il souhaiterait continuer à les exploiter (article 38). Si «chaque communauté, une fois obtenue son titre, pourra initier avec l'appui technique et matériel de l'OTR, l'étape d'assainissement de ses terres » (article 59), le texte de loi est en revanche remarquablement discret sur les modalités concrètes d'application de cet article et les moyens financiers à disposition des communautés pour indemniser les tiers.

\section{2 À Tasba Pri : un renforcement du pouvoir des élites locales, sans rétrocession de terres aux familles pauvres}

Votées en 2003, les étapes de résolution des conflits et de bornage prévues par la loi 445 ne commencent réellement à Tasba Pri qu'en 2011, alors que l'avancée du front pionnier traduit une colonisation métisse achevée de ce territoire et que les autorités miskitues de la plupart des villages ont progressivement vendu un droit d'usage pour toutes les terres communales.

Le «territoire indigène de Tasba Pri » est désormais découpé en dix zones, représentée chacune par une "communauté indigène de référence », administrée par une Assemblée communale dirigée par un bureau d'élus communaux aux fonctions diverses, dont un responsable de l'administration des ressources et des terres (appelé sindico). Les travaux de Moreno Blanco et al. (2012) permettent de reconstituer 1'histoire foncière de ces différentes communautés. Quatre de ces zones coïncident chacune avec l'un des villages miskitus fondés dès la fin des années 1980 et qui disposaient déjà d'un titre communal de réforme agraire : d'une part les villages implantés sur d'anciens camps (Sahsa, Sumubila) où les leaders indigènes ont fait jouer leurs appuis politiques pour agrandir largement au passage le territoire de leur communauté, qui inclut donc désormais d'autant plus de familles métisses ; d'autre part les villages implantés par les familles arrivées dès les années 1960, déplacées puis revenues dans la région (Kuakuil II, Altamira), qui ont conservé le strict finage qui leur avait été attribué à l'époque. Cinq autres zones ont été délimitées autour de villages formés plus tardivement sans titre communal de réforme agraire : Kukalaya, dont $75 \%$ de la population est aujourd'hui métisse, Akawasito, El Naranjal, Nazareth et San Miguel, villages désormais quasi exclusivement peuplés de Métis auxquels les autorités territoriales ont néanmoins accordé le statut de communauté de «matrice indigène ». En déployant des stratégies opportunistes de valorisation de leur affiliation indigène [Bataillon, 2000] (affiliation miskitue par union ou par ascendance lointaine), les leaders métis de ces quatre communautés sont ainsi parvenus à disposer, au nom du village, d'un titre de propriété sur de vastes espaces. Classée comme réserve protégée, la dernière de ces zones (Colombus) doit à son enclavement et aux lucratives activités d'orpaillage d'être aujourd'hui une zone 


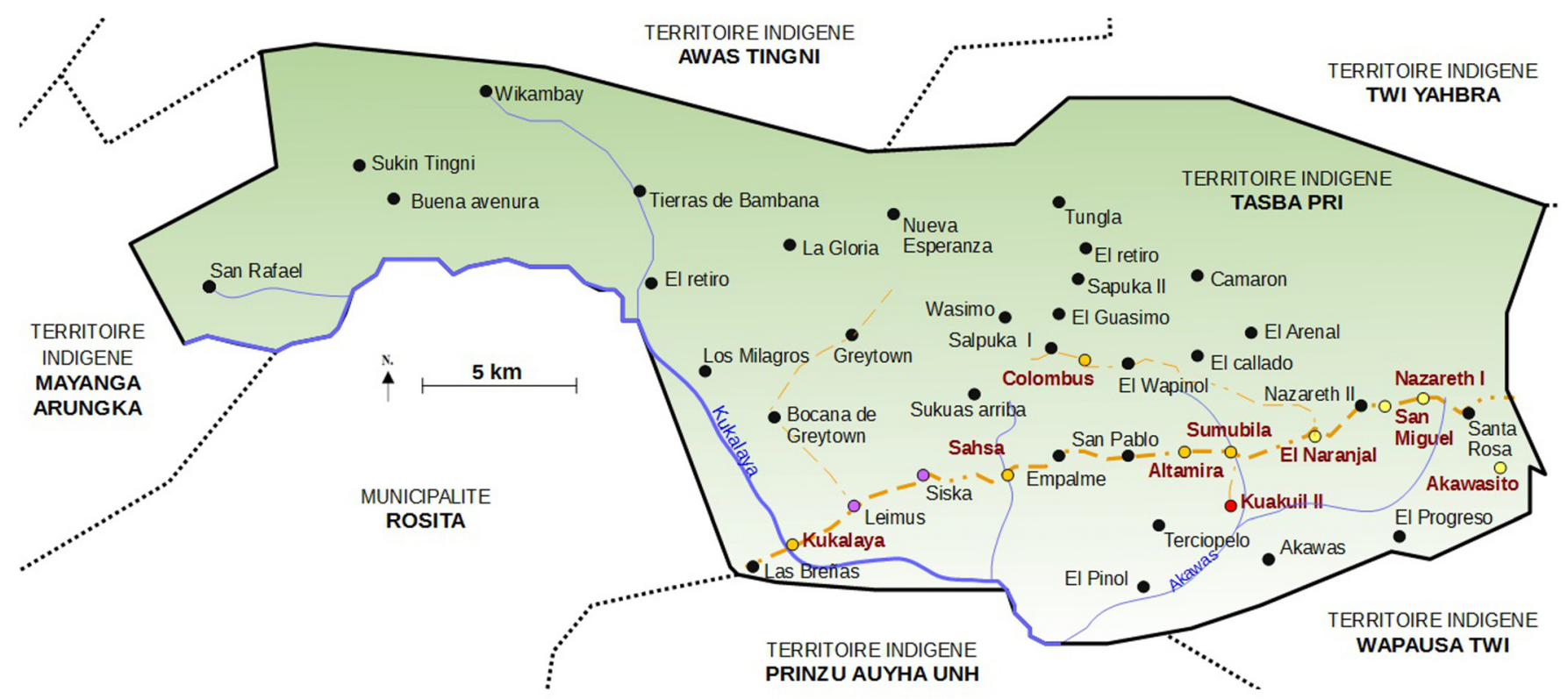

Communautés déclarées en 2011 de « matrice indigène »

Colonies subordonnées aux communautés de « matrice indigène »

- Villages de peuplement initial et actuel indigène

- Villages de peuplement initial indigène et aujourd'hui à prédominance métis de l'ouest

- Villages de peuplement initial métis natifs de la RACCN (lien de parenté miskitu) aujourd'hui à prédominance métis de l'ouest (pré-conflit)

- Colonies de peuplement métis ( $<25$ ans)

- Villages de peuplement initial métis natifs de la RACCN (lien de parenté miskitu) aujourd'hui à prédominance métis de l'ouest (préconflit) qui n'ont pas obtenu le statut de «matrice indigène ».

... Axes routiers

Rivières et Fleuves

Fig. 3. Occupation actuelle du Territoire indigène de Tasba Pri : statut et peuplement des villages. Réalisation : Leo Florent; source : enquêtes.

Fig. 3. Current occupation of the Tasba Pri indigenous territory: status and settlement of villages.

de non-droit. Pour des raisons de sécurité, elle n'a pas pu être étudiée de façon plus approfondie (Fig. 3).

$\mathrm{Au}$ sein des villages fondés dès les années 1980 par des Miskitus, les migrants métis ont le plus souvent payé pour obtenir les terres communales sur lesquelles ils se sont installés (sommes détournées à l'époque par les représentants des autorités villageoises qui leur avaient servi d'intermédiaires). Ils n'envisagent donc pas de partir, d'autant que l'absence de titre foncier actant la transaction ne leur permet de prétendre à aucune indemnisation, que les communautés indigènes seraient d'ailleurs bien en peine de leur verser. Aujourd'hui numériquement largement majoritaires dans ces villages référencés comme «indigènes », ils n'acceptent pas de devoir verser le loyer qu'exigent les autorités villageoises miskitues nouvellement élues (sindico) en vertu de l'article 38 de la loi 445 .

Nos enquêtes montrent par ailleurs que, face à la volonté d'agrandissement des éleveurs métis, la loi 445 n'a pas permis de mettre fin au processus de revente de droits d'usage sur les terres en territoire déclaré «indigène ». Sans ressources financières qui permettraient $d$ 'indemniser les agriculteurs métis pour les aménagements réalisés sur les parcelles dont la loi 445 prévoit la rétrocession aux communautés indigènes, les autorités indigènes ont fait le choix d'autoriser la poursuite des transferts fonciers pour les éleveurs qui souhaiteraient partir s'installer ailleurs. Ces transactions sont basées sur la vente d'un droit d'usage des terres, en prélevant au passage une commission fixée à $10 \%$, qui revient à chaque Assemblée communale. Elles sont maquillées par le versement d'une indemnisation des «améliorations » réalisées par le cédant, comme le stipule l'article 37 qui prévoit, lui, une rétrocession à la communauté indigène concernée et non à un nouveau « tiers ». Celles des familles miskitues qui en sont réduites à exploiter en fermage de très faibles surfaces n'ont pas les moyens de s'aligner sur ces tarifs, de 150 à 700 dollars l'hectare en fonction de la localisation et du degré d'aménagement des parcelles, et n'ont pas non plus le poids politique pour faire valoir leurs droits au sein de l'Assemblée communale ou de l'autorité territoriale de Tasba Pri.

Enfin, dans les communautés qui ont bénéficié d'un élargissement de leur finage, certains leaders indigènes profitent de l'argument de l' « assainissement » pour expulser des éleveurs familiaux métis installés sans autorisation, afin de revendre le droit d'usage de ces terres à leur seul profit et prolonger ainsi leur rente de situation, renforçant le ressentiment des agriculteurs métis de la région et contribuant à accroître les tensions entre Métis et Miskitus. Le renforcement du pouvoir des leaders indigènes prévu par la loi 445 n'est donc pas nécessairement synonyme d'une plus grande équité dans l'accès à la terre pour les familles indigènes. Des contre-exemples existent néanmoins, à l'image de Kuakuil II, où depuis près de 30 ans les villageois miskitus et leurs autorités ont eu à cœur de conserver l'usage de l'ensemble de leurs terres communales, ne procédant qu'exceptionnellement à la vente de terre pour financer des travaux 
d'intérêt général, et où s'observe aujourd'hui une différenciation sociale entre familles indigènes bien moins marquée.

\section{Conclusion}

À Tasba Pri, région aujourd'hui totalement gagnée par ce front pionnier, le flux continu de population et l'intégration croissante aux échanges marchands ont conduit à l'émergence d'une élite indigène qui s'est enrichie grâce à la vente illégale de droits d'usage sur les terres et ressources naturelles. Le capital accumulé par ces élites leur a permis de se lancer dans des productions plus rémunératrices et d'accroître leur surface cultivée en embauchant de la maind'œuvre et en développant des systèmes patronaux. Au sein de cette agriculture dont le caractère strictement manuel limite l'accroissement de la productivité du travail, la rentabilité et la pérennité de ces systèmes patronaux reposent aussi sur des salaires agricoles bas, que favorise la paupérisation des familles indigènes spoliées de leurs terres et obligées de louer leurs bras pour survivre, ou de migrer vers les ceintures de pauvreté des nouveaux centres urbains (Zeledon, 2005). Ces inégalités intra-ethniques se manifestent avec la même ampleur entre producteurs métis, où les derniers arrivés s'installent sur des terres bien moins favorables à l'élevage et travaillent en complément chez des éleveurs patronaux présents dans la région parfois depuis près de 20 ans.

L'éclatement de cette agriculture entre des systèmes de production familiaux en voie de paupérisation et des systèmes patronaux se traduit par des conflits dans l'accès au foncier de plus en plus violents, que l'application de la loi 445 dans la région ne parvient pas à résoudre. Le cas de Tasba Pri montre que la latitude existe, pour les autorités indigènes qui souhaitent s'y livrer, d'instrumentaliser cette loi pour accroître leur pouvoir économique, en fragilisant au passage l'accès au foncier des familles métisses les moins riches sans sécuriser et renforcer celui des familles miskitues paupérisées.

Sans moyens financiers prévus pour que les communautés indigènes puissent indemniser les tiers afin de faciliter leur départ des terres indigènes qu'ils occupent, sans dispositif de réinstallation des éleveurs métis sur d'autres terres réellement «libres » et surtout sans s'attaquer aux causes de ces flux migratoires depuis le centre-ouest et à leur gestion à un niveau fédéral, la loi 445 supposée aider à la résolution des conflits dans les territoires déjà gagnés par le front pionnier semble pour l'instant se révéler peu opérante.

En parallèle, les installations de métis qui accompagnent l'avancée du front pionnier, bien souvent de simples métayers de propriétaires de grands troupeaux implantés en arrière de ce front, se poursuivent vers les derniers territoires forestiers plus au nord de la RACCN, avant la frontière hondurienne.

\section{Références}

Asamblea nacional de la República de Nicaragua. 1987. Estatuto de la autonomía de las regiones de la costa atlántica de Nicaragua. ley no. 28 de 7 de septiembre de 1987. La Gaceta 238 (30 de octubre de 1987).
Asamblea nacional de la República de Nicaragua. 2003. Ley de régimen de propiedad comunal de los pueblos indígenas y comunidades étnicas de las regiones autónomas de la costa atlántica de Nicaragua y de los ríos Bocay, Coco, Indio y Maíz. Ley no. 445, aprobada el 13 de diciembre del 2002. La Gaceta 16 (23 de enero del 2003).

Bainville S, Mena R, Rasse-Mercat E, Touzard L. 2005. La pauvreté des exploitations familiales nicaraguayennes : retard technique ou manque de terre? Revue Tiers-Monde 46(183): 559-580. DOI: $10.3406 /$ tiers.2005.5593.

Bataillon G. 2000. Moskitia nicaraguayenne : changements sociopolitiques et égalisation des conditions (1979-1999). In : Musset A, Delhom J, eds. Nicaragua dans l'œil du cyclone : en el ojo del huracan. Paris (France) : Éditions de l'IHEAL, pp. 120-134.

Boege E. 1987. La contradiction ethnie-nation : le cas des Miskitos au Nicaragua. Anthropologie et Sociétés 11(2): 49-63. DOI: 10.7202/ 006417ar.

Cochet H, Devienne S. 2006. Fonctionnement et performances économiques des systèmes de production agricole : une démarche à l'échelle régionale. Cahiers Agricultures 15(6): 578-583. DOI: http://sci-hub.cc/10.1684/agr.2006.0028.

Cochet H, Devienne S, Dufumier M. 2007. L'agriculture comparée : une discipline de synthèse ? Économie Rurale 297-298: 99-112. DOI: 10.4000 /economierurale.2043.

Fundación para el Desarrollo Tecnológico Agropecuario y Forestal de Nicaragua (FUNICA), Fundación Ford - Gobierno Regional. 2009. Caracterización socioeconómica de la Región Autónoma del Atlántico Norte (RAAN) de Nicaragua, 60 p.

Garcia Izaguirre PA. 2011. Conflictos territoriales en la comunidad de Wasakin, Municipio de Rosita, Región Autónoma Atlántico Norte (RAAN). Ciencia e Interculturalidad 9(2): 10-26.

Hardy S. 2005. Intégration pacifique et autonomie atlantique au Nicaragua (integration and autonomy in Nicaragua, Pacific versus Atlantic). In : Bret $\mathrm{B}$, ed. Intégration et fragmentation territoriale en Amérique latine. Bulletin de l'Association des géographes français, $82^{\mathrm{e}}$ année, 2005-4 (décembre), pp. 422-432.

Henriksen K, Kindblad C. 2005. El dilema colectivo de los pueblos miskitos en los años 90 . El caso de Tasba Pauni. WANI 42: 6-39.

Kinlochet Tijerino F. 1997. Conflicto étnico, geopolitica e identidad: el caso de las comunidades Miskitas del rio Coco en Nicaragua. Estudios Fronterizos 40: 95-114.

Klein JL. 1986. Défi au développement régional. Territorialité et changement social au Nicaragua sandiniste. Québec : Presses de l'université du Québec, 169 p.

Leo F. 2016. Diagnostic agraire du territoire multi-ethnique de Tasba Pri (côte caribéenne autonome Nord Nicaragua), Mémoire de DAA. Paris : AgroParisTech, $141 \mathrm{p}$.

Lévêque F. 1986. Les processus de formation et les dynamiques des régions pionnières. Les cas de la côte atlantique nicaraguayenne et de l'Amazonie brésilienne. Cahiers des Sciences Humaines 22(3-4): 345-354.

Maldidier C. 2004. Agricultural pioneer fronts, the crest of a farreaching wave. The social and spatial dimension of lowlands colonization in Nicaragua. In: Babin D, ed. Beyond tropical deforestation. Unesco-Cirad, pp. 185-192.

Moreno Blanco E, Müller Lacayo R, Chow Serapio K, Llezeth Moreno S, Avellan Rivera E, Mairena D, et al. 2012. Estudio de caso del territorio de Tasba Pri. In: Larson AM, Soto F, eds. Territorialidad in gobernanza. Tejiendo Retos en los Territorios Indígenas de la RAAN, Nicaragua. Instituto Nitlapan de la Universidad Centroamericana, pp. 119-176. 
Paul JL, Bory A, Bellande A, Garganta E, Fabri A. 1994. Quel système de référence pour la prise en compte de la rationalité de l'agriculteur : du système de production agricole au système d'activité. Les cahiers de la recherche-développement 39: 7-19.
Zeledon MR. 2005. Campesinos de la frontera agrícola. Sujetos sociales desconocidos e identidades negadas. In: Euraque DA, Gould JL, Hale CR, eds. Memorias del mestizaje, Cultura política en Centroamérica de 1920 al presente. CIRMA, pp. 565-577.

Citation de l'article : Leo F, Garambois N. 2017. Démarcation des terres indigènes et inégalités en zone de front pionnier au Nicaragua : le cas de Tasba Pri. Cah. Agric. 26: 35003. 